\title{
Design of $50 \mathrm{~Hz}$ notch filter based on virtual instrument
}

\author{
Fuhai HUANG ${ }^{1,}$, , Zhenhai Wang ${ }^{1}, \mathrm{Xu} \mathrm{Bo}^{2}$ \\ ${ }^{1}$ School of Informatics, Linyi Normal University, Linyi, 276000, China \\ ${ }^{2}$ School of Automobile Engineering, Linyi Normal University, Linyi, 276000, China \\ aemail: hfh8130@163.com
}

Keywords: LabVIEW, Notch Filter, $50 \mathrm{~Hz}$ power frequency interference

\begin{abstract}
Hz}$ power frequency interference is the main noise of ECG signal. It is harmful to automatic detection and the classification of arrhythmia. Therefore, it is essential to reject $50 \mathrm{~Hz}$ interference in the preprocess of ECG signal. This paper develops a $50 \mathrm{~Hz}$ notch filter based on LabVIEW. The notch filter can reject $50 \mathrm{~Hz}$ power frequency interference efficiently.
\end{abstract}

\section{Introduction}

In the acquisition process of biomedical signals, the ECG signal amplitude is very low, very susceptible to interference from the external environment, and the $50 \mathrm{~Hz}$ power frequency interference is the main interference source of ECG signal. Therefore, how to effectively suppress the $50 \mathrm{~Hz}$ interference is a long-term study of people's problems. In the solution of many of the most commonly used method, is simple, is to design a fixed center frequency band stop filter is a notch filter to eliminate the power line interference. And for $50 \mathrm{~Hz}$ in the design method of filter is varied, can be implemented by hardware, but the implementation of the process more difficult; method can also be used to realize the software, such as adaptive filtering, wavelet transform filtering method, this method can be realized by MATLAB, but MATLAB in the signal acquisition and preparation of GUI are poor, and the software of the operating environment requirements are relatively high, the software running speed, restricting the scope of software. "The software is the instrument" reflects the essential characteristics of virtual instrument technology. The concept of virtual instrument was first put forward in the development of LabVIEW, LabVIEW is one of the most successful and most widely virtual instrument software development environment. Therefore, notch filter based on virtual instrument software LabVIEW design company NI to eliminate frequency interference.

\section{The design of notch filter based on LabVIEW $50 \mathrm{~Hz}$}

Graphical virtual instrument integrated development environment LabVIEW (Laboratory Virtual Instrument Engineering Workbench) is a software development platform of virtual instrument NI company, using the graphical programming language, the program block diagram form, easy to learn and easy to use, can support and expression analysis to source level device its intuitive and easy programming, many the driver, a variety of, have created the basis of conditions for users to quickly and easily build instrument system their own needs in practical production. Compared with the LabVIEW and other computer languages, has a particularly important difference: the other computer languages are used to generate code for text based on LabVIEW language, using the graphical programming language.

In the digital signal processing toolbox of LabVIEW provides a number of tools, filters can design all kinds of performance is very convenient. The filter type is divided into infinite impulse response filter (IIR) and the finite impulse response filter (FIR) of two, the former is characterized by the order of the filter required is relatively low, small amount of calculation, but also destroys the phase characteristics, waveform distortion. While the use of FIR filter can get better filtering effect, also can make the waveform distortion is minimized, and the FIR filter can be made into a linear phase characteristic, which is required for the filtering of ECG signal. Compared with the IIR filter, FIR filter order also requires very high performance, the computation is much larger, but the current 
situation and its configuration, this is no problem.

Many methods has been provided, such as: the design method of FIR filter Chebyshev approximation method, sampling method and the window function, the LabVIEW provides a direct filter design tools, design only needs to set a specific filter parameters can be realized, and the calculation of parameters also have the corresponding windowed FIR filter coefficients to achieve. This paper uses the window function method to design $50 \mathrm{~Hz}$ filters. The first requirement of window function method to choose a proper ideal low-pass filter, because the impulse response is non causal and infinite impulse response, to intercept it using optimization window window function, so as to obtain the linear phase FIR filter and causality. In a number of window function, Kaiser window is the window function is close to the optimal window structure, it can adjust the filter parameters according to the different, so using the Kaiser window function in the filter design. In LabVIEW, available MathScript node to describe the Kaiser window format is: C = Kaiser (a, B). This function is to produce a Kaiser window. The function a is Kaiser the length of the window, B is a special reaction parameters, the relationship between the window function of the main lobe width and the side lobe attenuation, the return value is C. In the FIR window coefficient tool in Figure 1 the window parameter is used to select the window function, taps is used to set the number of taps, Kaiser window length a, window the parameter parameter is used to set the Kaiser window B. At the same time, we should choose the type of filter is a low-pass filter and the entrance of its parameters, low cut-off frequency and sampling frequency. Filter generation tool is shown in figure 1.

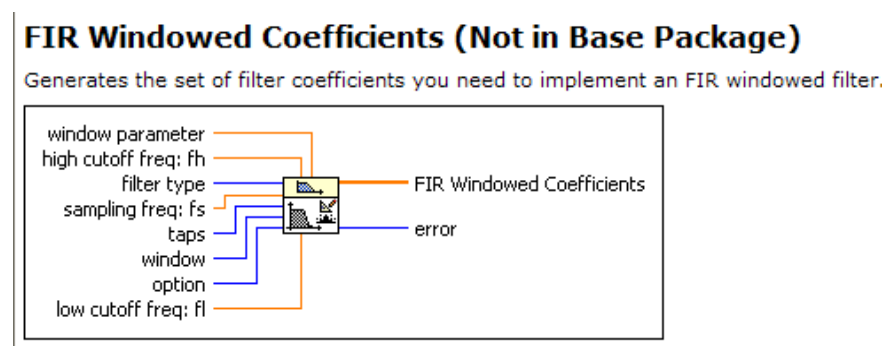

Fig.1. FIR window coefficient generation tool

Notch filter is usually caused by a low pass filter and high pass filter composed of the last, in order to eliminate the interference of $50 \mathrm{~Hz}$, set the high pass filter cutoff frequency for $51 \mathrm{~Hz}$, low pass filter cutoff frequency is set to $49 \mathrm{~Hz}$. Thus we can get the parameters of FIR filter, connect the figure 2 to complete the design of notch filter.

\section{FIR Filter (DBL)}

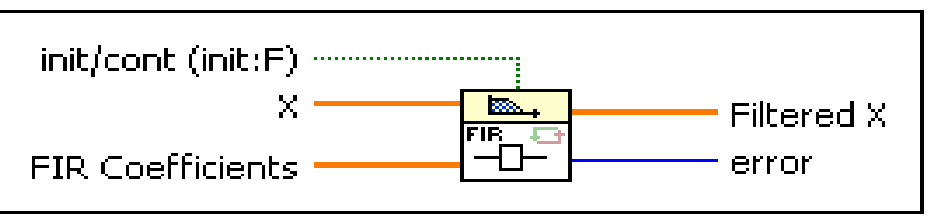

Fig. 2. FIR filter design tool

The disturbance of $50 \mathrm{~Hz}$ diagram of notch filter LabVIEW implementation is shown in figure 3 .

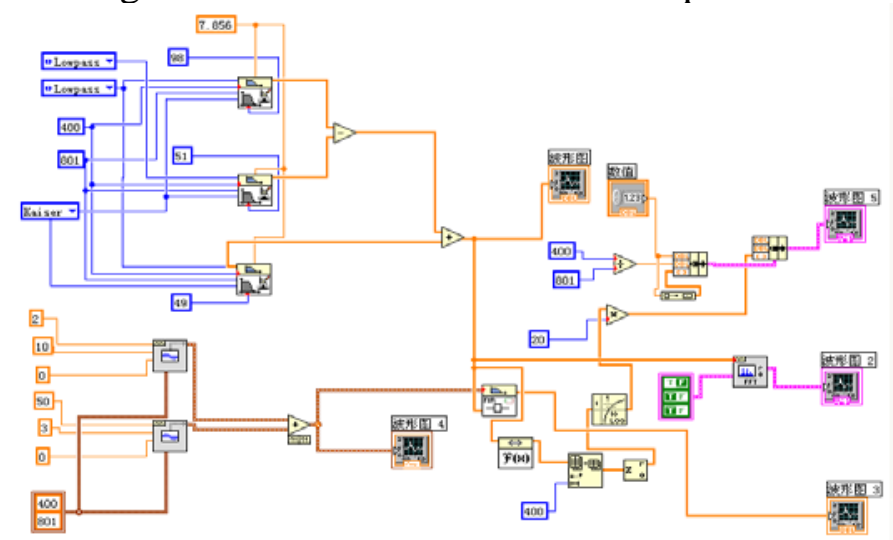

Fig.3. Notch filter control flow chart 


\section{Analysis of the results}

The amplitude frequency characteristics of the notch filter and the impulse response as shown in Figure 4, the amplitude frequency characteristics can be seen in the picture, the notch filter in the passband attenuation and magnitude is not very smooth, at the center frequency of $50 \mathrm{~Hz}$, the attenuation of close to $40 \mathrm{~dB}$.
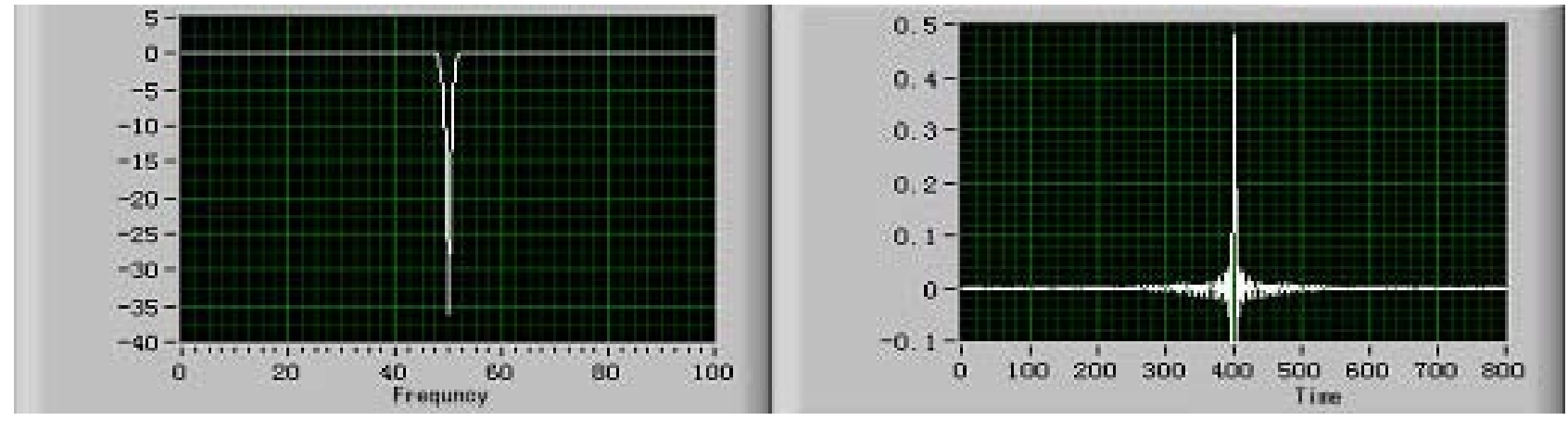

Fig.4. The amplitude frequency characteristic and the impulse response

The phase characteristics of Figure 5, the signal in the frequency range, in meet the linear phase filter.

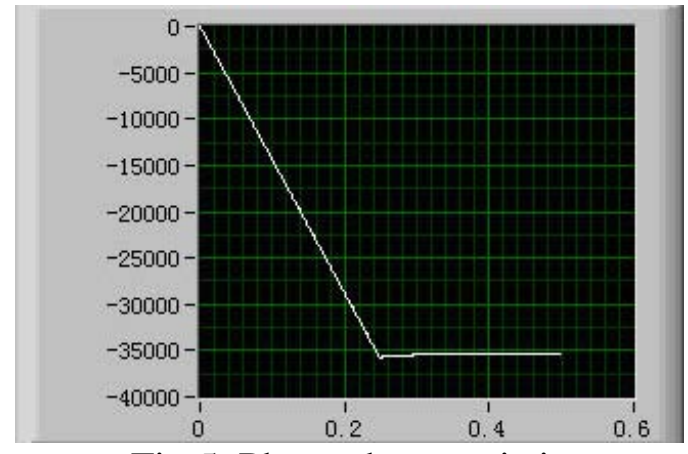

Fig.5. Phase characteristic

\section{The example simulation}

Assume that the input signal $f(t)=10 \sin 4 \pi t+3 \sin 100 \pi t$ as the original input signal, the sampling frequency is set to $400 \mathrm{~Hz}$, by comparing before and after the filter graph as shown in figure 6 . The output waveform characteristics, effectively inhibit the $50 \mathrm{~Hz}$ frequency interference, it is verified that the design methods of the notch filter is ideal.

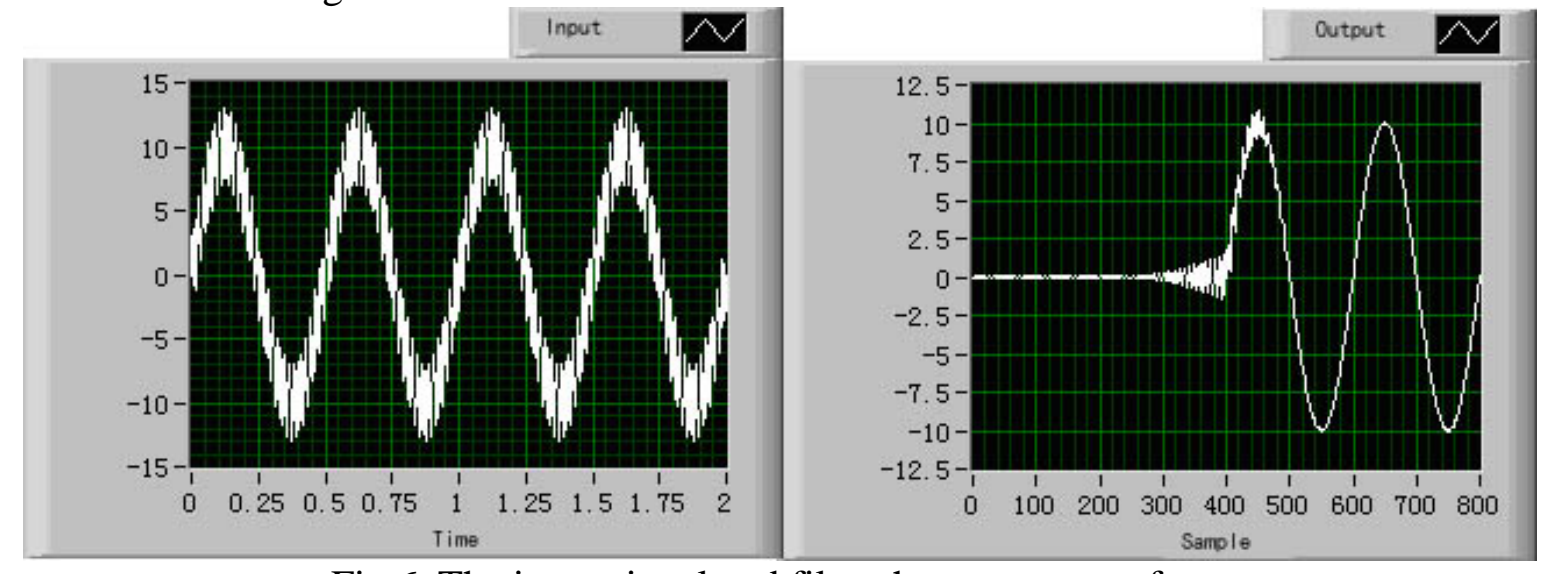

Fig.6. The input signal and filter the output waveform 


\section{Conclusion}

This paper designed the notch frequency interference based on LabVIEW, and proves its good performance, can be used for the power line interference suppression of $50 \mathrm{~Hz}$, the input signal can be acquired by data acquisition card, and combined with the real system in engineering and the actual use is to have certain practical significance.

The author of this paper innovation points: the use of virtual instrument design of power frequency notch filter, the virtual instrument with good interface and powerful function of data acquisition, processing can be easily applied to the practical signal.

\section{References}

[1] Yang Leping, Li Haitao. Design and application of LabVIEW. Publishing House of electronics industry, 2005

[2] He Yujun, Gao Huisheng. LabVIEW virtual instrument design. The posts and Telecommunications Press, 2012

[3] Tian Hao, Duan Lijun. Design of multi function digital filter based on LabVIEW. The electronic measurement technology, 2011

[4] Joerg Christian Wolf, Phil Hall, Paul Robinson, Phil Culverhouse. Bioloid based Humanoid Soccer Robot Design, 2007.

[5]Wu Chuan-yu, He Lei-ying, Design and Realization of Instructional RPPR-Robot, Research and Exploration in Laboratory.2007, 26(10) 